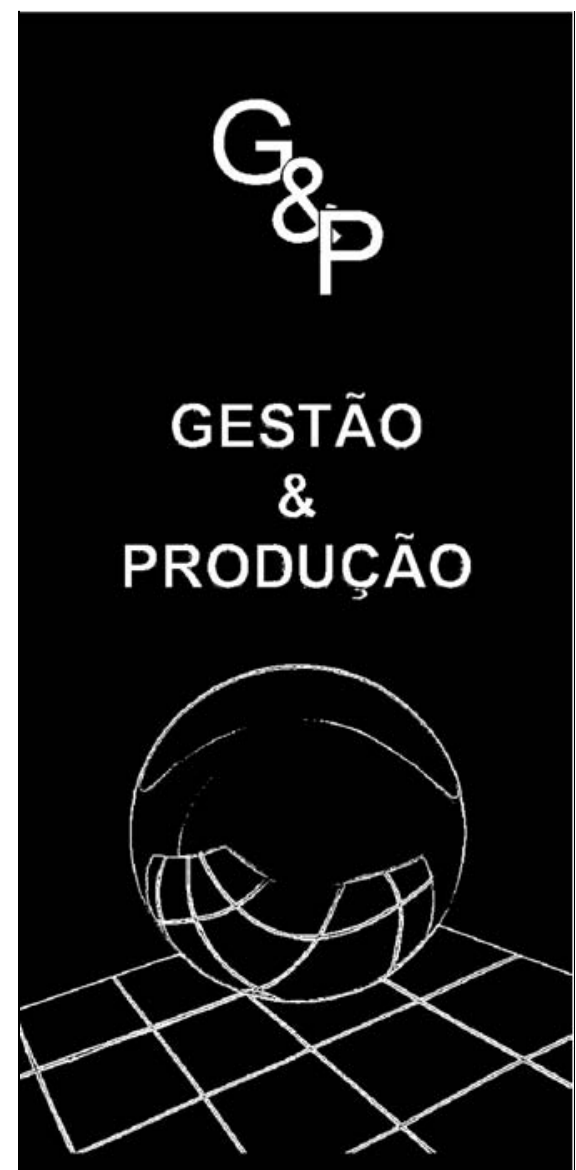

v.5, n.1, p. 69-80, abr. 1998

\section{MODELAGEM DE SISTEMAS DE MANUFATURA FLEXÍVEIS CONSIDERANDO RESTRIÇÕES TEMPORAIS E A CAPACIDADE DO MAGAZINE}

\author{
Arthur Tórgo Gómez \\ UNISINOS - Centro de Ciências Exatas \\ Caixa Postal 275 - 93022-000 - São Leopoldo - RS \\ INPE \\ Caixa Postal 515 \\ 12201-970 - São José dos Campos - SP \\ Luiz Antonio N. Lorena \\ LAC/INPE \\ Caixa Postal 515 \\ 12201-970 - São José dos Campos - SP
}

\title{
Resumo
}

Neste trabalho é apresentado um modelo que gera o seqüenciamento de partes e carregamento de ferramentas em um ambiente de manufatura flexível formado por uma máquina. São consideradas datas de vencimento das partes a serem processadas, os períodos dos turnos de produção, e uma restrição física de capacidade do magazine que armazena as ferramentas necessárias ao processamento das partes. No desenvolvimento do modelo são abordados os problemas de seleção de partes, de carregamento de ferramentas e o problema de "scheduling" com restrições. Um seqüenciamento inicial das partes e carregamento de ferramentas é obtido usando um algoritmo para identificação de grupos que considera a capacidade do magazine. A solução inicial é então melhorada pelo uso de busca tabu, gerando seqüencias de partes e carregamento de ferramentas que refletem políticas de otimização determinadas por pesos em uma função objetivo. Vários testes foram realizados para validação do modelo, sendo aqui apresentados alguns resultados obtidos considerando os problemas de minimização do número de trocas de ferramentas, do número de instantes de parada para a troca de ferramentas, do tempo de atraso e do tempo referente ao período ocioso dos turnos de produção.

Palavras-chave: seleção de partes, carregamento de ferramentas, scheduling. 


\section{Introdução}

$\mathrm{N}$ este estudo é proposto um modelo para gerar o seqüenciamento de partes $e$ carregamento de ferramentas em um ambiente de manufatura flexível formado por uma máquina, considerando datas de vencimento das partes a serem processadas, turnos de produção, e a restrição física imposta pela capacidade limitada do magazine que armazena as ferramentas necessárias ao processamento das partes.

No desenvolvimento do modelo são abordados os problemas de seleção de partes (STECKE \& KIM, 1988 e 1991; HWANG \& SHOGAN, 1989; e GYAMPAH, 1994), de carregamento de ferramentas (HIRABAYASHI et al., 1984; TANG \& DENARDO, $1987 \mathrm{e}$ 1988; BARD, 1988; STECKE \& KIM, 1988; STECKE, 1989; JAIKUMAR \& VAN WASSENHOVE, 1989; HWANG \& SHOGAN, 1989; STECKE \& TOCZYLOWSKI, 1992; FOLLONIER, 1994; MORENO \& DING, 1993; CRAMA et al., 1994; e GYAMPAH, 1994) e o problema de "scheduling" com restrições (BLAZEWICZ et al., 1991; HE \& KUSIAK, 1992; e BLAZEWICZ \& FINKE, 1994). Um seqüenciamento inicial de partes e carregamento de ferramentas é obtido usando um algoritmo para identificação de grupos que considera a capacidade do magazine. A solução inicial é então melhorada pelo uso de busca tabu, gerando seqüências de partes e carregamento de ferramentas que refletem políticas de otimização determinadas por pesos em uma função objetivo.

$\mathrm{Na}$ seção 2 apresenta-se uma definição do sistema de manufatura flexível (SMF) a ser estudado, com as suposições necessárias ao desenvolvimento do modelo de seleção de partes $e$ de ferramentas (MSPF). O MSPF é discutido na seção 3. Os resultados obtidos em testes computacionais são descritos na seção 4 , e as conclusões do estudo realizado são apresentadas no final do artigo.

\section{O Sistema de Manufatura Flexível (SMF)}

$\mathrm{U}$ m SMF é formado por um conjunto de máquinas versáteis e um sistema automático de manejo de materiais, capazes de processar simultaneamente volumes pequenos e médios de uma variedade de tipos de partes, controlados por um sistema computacional e por operadores (JAIKUMAR \& VAN WASSENHOVE, 1989; STECKE, 1989; STECKE \& TOCZYLOWSKI, 1992; KOUVELIS, 1992; e OERLEMANS, 1994).

Neste estudo, o ambiente de manufatura flexível considerado é formado por uma máquina versátil, capaz de processar qualquer parte que lhe seja destinada, desde que as ferramentas adequadas estejam em seu magazine no instante de processamento da parte. Considerando um sistema formado por várias dessas máquinas, as ferramentas a elas designadas determinam suas diferenças funcionais. Dado que todas as máquinas possuem a mesma flexibilidade de máquina é possível tratar o sistema inteiro como se fosse formado por uma só máquina.

Um lote é um conjunto de partes que podem ser processadas continuamente sem exceder o período do turno de produção ao qual pertencem. A palavra continuamente significa que todas as partes do lote são processadas por um mesmo conjunto de ferramentas que se encontram no magazine da máquina no momento do processamento, e portanto, durante todo o processamento não são realizadas trocas de ferramentas.

Toda vez que termina o processamento de um lote, dispositivos de transporte são acionados, a área de trabalho é limpa, manutenções são realizadas, ferramentas são descarregadas e carregadas do magazine e dispositivos são preparados para o reinício do processamento. Este tempo de preparação entre o fim e o início de processamento de dois lotes é chamado de tempo de setup. As ferramentas e as partes são transportadas por um sistema automático de manejo de materiais do almoxarifado até a área de trabalho. Tanto a máquina como o manejo são controlados por um sistema computacional. 


\section{Descrição do MSPF}

S eja um conjunto de partes a serem processadas em um máquina versátil e um conjunto de ferramentas disponível para processá-las. Para cada parte supõe-se conhecidos seu tempo de processamento, sua data de vencimento e o comjunto de ferramentas necessárias para processá-la.

O MSPF considera ainda as seguintes condições:

- Cada parte requer um conjunto de ferramentas.

- A máquina possui um magazine com capacidade limitada. Esta restrição é crítica, pois restringe o número de ferramentas que podem ser carregadas e, conseqüentemente, $o$ número de partes que podem ser produzidas continuamente.

- Não existe loop no sistema. Uma parte que começa a ser processada é finalizada e não retorna. Isto significa que todas as partes são processadas e que o conjunto de ferramentas necessário para processar cada parte deve estar no magazine antes do início do processamento.

- Os tempos de setup são considerados separados dos tempos de processamento das partes, sendo constituídos por dois períodos: pelo período de preparação do sistema para reinício de processamento (preparação da máquina e de dispositivos, limpeza da área de trabalho e manutenções) e pelo período de tempo gasto na troca de ferramentas. O tempo de preparação do sistema para o reinício de processamento é considerado fixo. O tempo de troca de ferramentas é considerado proporcional ao número de ferramentas trocadas.

- Os turnos de produção são respeitados; no momento de término de um turno, nenhuma parte pode estar em processamento ou vir a ser processada.

Para uma dada sequiência $s_{i}$ de partes a serem processadas, a função objetivo a ser minimizada é definida pela soma dos seguintes termos:

$$
\begin{array}{r}
f\left(s_{i}\right)=\mathrm{P}_{1} \cdot T p\left(s_{i}\right)+\mathrm{P}_{2} \cdot A t\left(s_{i}\right)+\mathrm{P}_{3} \cdot S p\left(s_{i}\right)+ \\
+\mathrm{P}_{4} \cdot S f\left(s_{i}\right)+\mathrm{P}_{5} \cdot \operatorname{Tr}\left(s_{i}\right)
\end{array}
$$

onde:

- $T p\left(s_{i}\right)$ é o tempo total de produção (makespan): instante em que a última parte da seqüência tem seu processamento finalizado;

- $\operatorname{At}\left(s_{i}\right)$ é o tempo total de atraso: referente ao somatório das diferenças positivas entre as datas de saída da produção de cada parte e suas respectivas datas de vencimento;

- $S p\left(s_{i}\right)$ é o tempo total de setup: preparação do sistema cada vez que o processamento é parado para a troca de ferramentas;

- $S f\left(s_{i}\right)$ é o tempo total de setup: troca de ferramentas;

- $\operatorname{Tr}\left(s_{i}\right)$ é o tempo total ocioso dos turnos: somatório das diferenças entre as datas de finalização dos turnos e as datas de saída de suas últimas partes processadas.

Associa-se um peso a cada parcela da função objetivo (respectivamente, $\mathrm{P}_{1}, \mathrm{P}_{2}, \mathrm{P}_{3}, \mathrm{P}_{4}$ e $\mathrm{P}_{5}$ ). Conforme os valores definidos para esses pesos, a função objetivo poderá refletir várias estratégias. O MSPF é então resolvido em duas fases: na primeira é gerada uma sequiência inicial de partes e na segunda a busca tabu (GLOVER \& LAGUNA, 1995) é empregada, obtendo-se sequiências de partes e carregamento de ferramentas que otimizam a estratégia definida pelos valores dos pesos atribuídos a função objetivo.

\subsection{Solução do MSPF - Primeira Fase}

Nesta fase obtém-se uma seqüência inicial $s_{0}$ de partes e seu respectivo carregamento de ferramentas. Os lotes das partes são identificados como conjuntos de partes vizinhas em $s_{0}$ que podem ser processadas por um mesmo conjunto de ferramentas. Dentro de cada lote as partes são seqüenciadas considerando suas datas de vencimento.

Para a identificação dos lotes usamos uma versão modificada do algoritmo de identificação de grupos, proposto por KUSIAK \& CHOW (1986). Um algoritmo modificado de identificação de grupos (AMIG), derivado deste algoritmo, foi proposto por GÓMEZ (1992) levando em conta a capacidade limitada do magazine. A 
aplicação do algoritmo modificado em uma matriz do tipo partes $x$ ferramentas permite obter os grupos de partes e seus grupos de ferramentas associadas, tal que a capacidade do magazine sempre é respeitada.

Como exemplo de aplicação, suponha a matriz $\mathrm{A}: \mathrm{a}_{\mathrm{ij}}=1$, se a ferramenta $\mathrm{j}$ é necessária para o processamento da parte $i$, e $a_{i j}=0$, caso contrário.

\section{Ferramentas}

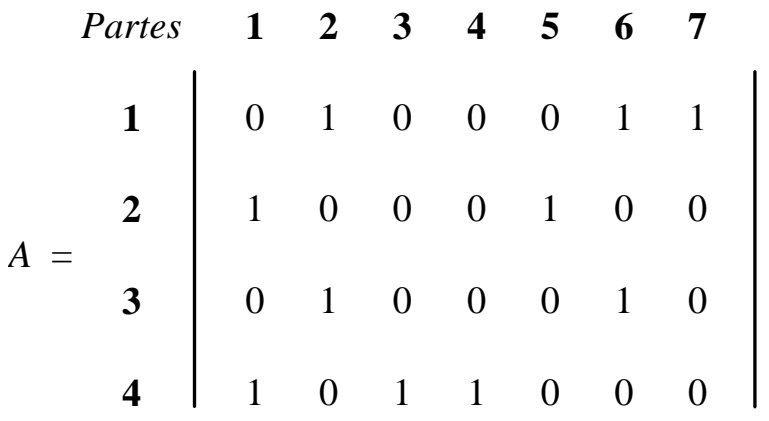

Com a aplicação do algoritmo de Kusiak e Chow, obtém-se:

\section{Grupos de Ferramentas}

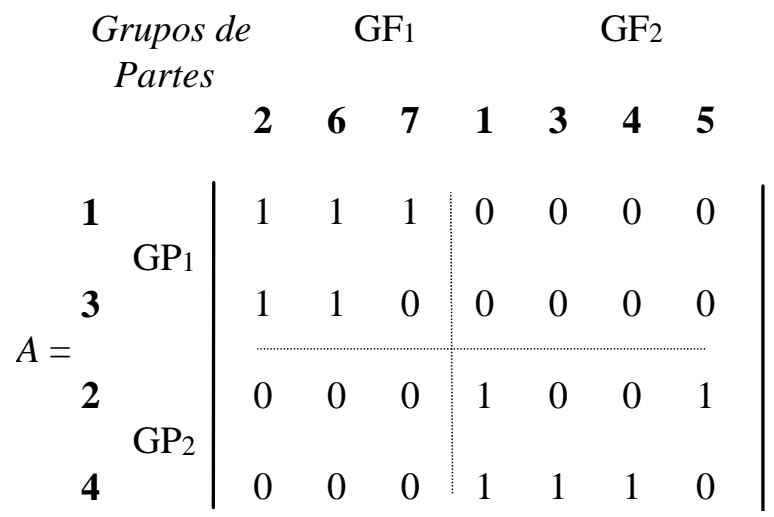

Supondo em três a capacidade do magazine de ferramentas, a solução do algoritmo não seria válida, pois as partes 2 e 4 que formam o segundo grupo de partes, não poderiam ser processadas com um mesmo conjunto de ferramentas. $\mathrm{O}$ algoritmo modificado considera o mesmo algoritmo de formação de grupos, mas impede que grupos de ferramentas excedam a capacidade do magazine (maiores detalhes estão em GÓMEZ (1996)). Sua aplicação nos mesmos dados fornece:

\section{Grupo de Ferramentas}

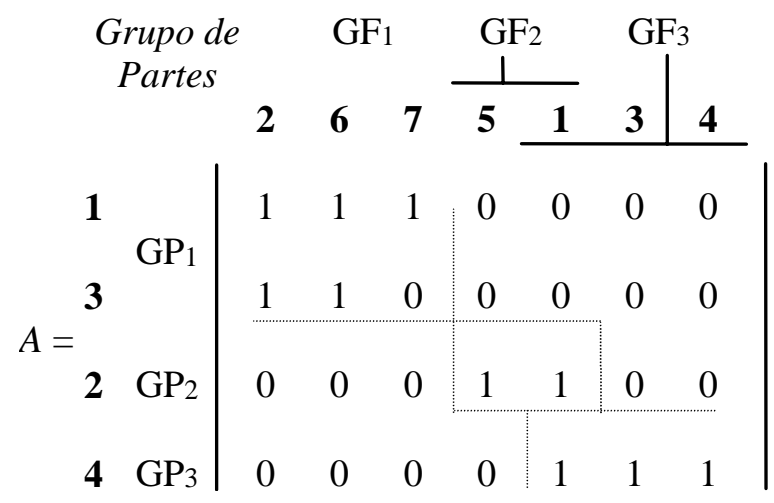

A seqüência inicial identificada $s_{0}=(1,3,2,4)$ poderá produzir os lotes $(1,3),(2)$ e (4) se os períodos dos turnos de produção forem respeitados. O lote $(1,3)$ é ainda sequienciado internamente considerando as datas de vencimento de suas partes, podendo ser considerado como $(3,1)$ caso seja a melhor opção com a consideração do atraso. A sequiência $s_{0}=(1,3,2,4)$ será então reescrita como $s_{0}=((1,3),(2),(4))$, onde os lotes são destacados.

\subsection{Solução do MSPF - Segunda Fase}

Nesta fase a busca tabu será empregada para reduzir o número de conflitos entre o cumprimento das datas de vencimento das partes e o respeito à designação de partes aos lotes. $\mathrm{O}$ conflito pode ser descrito pela seguinte situação: uma parte ocupa uma dada posição na seqüência de processamento de tal forma que não gera atraso. Seu posicionamento, porém, irá gerar duas paradas para a troca de ferramentas. $\mathrm{O}$ conjunto de ferramentas existente no magazine não permite processá-la e após o seu processamento a nova configuração do magazine não permite processar a próxima parte. Com o uso da busca tabu, tem-se a flexibilidade de, a partir de uma mesma solução inicial, gerar diferentes seqüências de partes e carregamento de ferramentas que 
retratam estratégias de otimização adotadas no gerenciamento do sistema.

A busca tabu tem sido usada com grande sucesso nos problemas reconhecidamente difíceis da Otimização Combinatória. Particularmente em problemas de "scheduling". Veja alguns dos artigos de Fred Glover (por exemplo, GLOVER \& LAGUNA, 1995) para uma descrição formal da busca tabu. A seguir é descrito o algoritmo tabu para seleção e "scheduling" de partes e carregamento de ferramentas.

\section{Algoritmo TSS}

$s_{0}=$ seqüência inicial;

$f_{-}$melhor $=f\left(s_{0}\right)$;

$f \_$atual $=f \_$melhor;

niter $=0 \quad$ \{número de iterações sem melhora\};

melhiter $=0$ \{número da iteração da melhor solução encontrada\};

nbmax $=20$ \{número máximo de iterações sem obter uma melhora na solução\};

Inicializar as listas tabu para duas buscas, $L_{l}$ e $L_{2}$.

Inicializar a função do critério de aspiração, $\operatorname{Asp}\left(f\left(s_{0}\right)\right)$;

$s=s_{0}$,

Enquanto (niter - melhiter $<$ nbmax) faça $f \_$atual $=f \_m e l h o r$;

niter $=$ niter +1 ;

\{Primeira busca de vizinhança\}

Gerar um conjunto $V_{l}$ de seqüências $s_{i}$ em $N_{l}$

Escolher a melhor sequiência $s_{i}^{+}$em $V_{l}$ que não é tabu ou $f\left(s_{i}^{+}\right)<A s p(f(s))$;

Atualize a lista tabu $L_{l}$ e a função critério de aspiração $\operatorname{Asp}(f(s))$;

Se $f\left(s_{i}^{+}\right)<f_{-}$melhor

fim_se

então $\quad f_{-}$melhor $=f\left(s_{i}^{+}\right)$,

$s=s_{i}^{+}$;

\{Segunda busca de vizinhança\}

Gerar um conjunto $V_{2}$ de sequiências $s_{i}$ em $N_{2}$

Escolher a melhor sequiência $s_{i}^{+}$em $V_{2}$ que não é tabu ou $f\left(s_{i}^{+}\right)<A s p(f(s))$;
Atualizar a lista tabu $L_{2}$ e a função critério de aspiração $A s p(f(s))$;

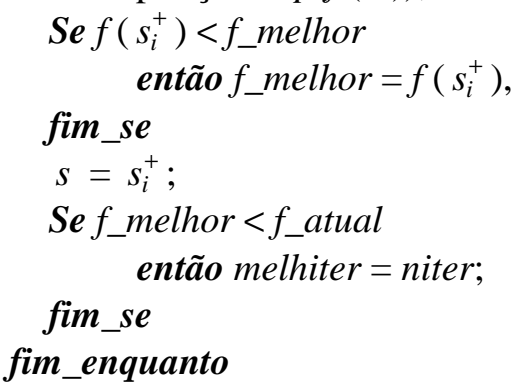

A cada iteração do algoritmo TSS são realizadas duas buscas de vizinhança $\left(N_{l}\right.$ e $\left.N_{2}\right)$. Em $N_{l}$, dada a seqüência de partes vigente na iteração anterior, uma permutação de dois lotes é verificada. Seja a seqüência $s_{0}=((1,3),(2),(4))$ identificada no exemplo da fase I, uma sequiência $s_{i}{ }^{+}$poderia ser dada nesse caso por ${s_{i}}^{+}=$ $((2),(1,3),(4))$. Os lotes contudo continuam inalterados. Em $N_{2}$, a partir de $s=((2),(1,3),(4))$, uma parte passa a ser processada em outro lote por movimentos de retirada e de inserção. No nosso exemplo uma sequiência possível iria quebrar o lote $(1,3)$, gerando por exemplo $s_{i}{ }^{+}=$ ((2),(1),(4),(3)). A formação de novos lotes deve sempre obedecer a capacidade do magazine. Cada uma das buscas de vizinhança possui uma lista tabu própria guardando as respectivas seqüências visitadas. O tamanho das listas foi definido de modo a evitar que a busca ficasse presa a mínimos locais. O critério de nível de aspiração utilizado nas duas buscas foi o mais comum: caso o movimento escolhido for tabu, mas o valor de sua solução for menor que a melhor solução vigente na busca tabu ( $f_{-}$melhor) o movimento é aceito.

Foram usadas duas vizinhanças para não se prender a uma definição inicial dos lotes, o que é obtido na inserção de partes, e também para melhorar os atrasos quando se faz uma troca de posição dos lotes na seqüência. Como vamos verificar nos testes computacionais descritos na próxima seção, os pesos na função objetivo contribuíram para a flexibilidade no modelo, considerando-se diversas políticas de otimização. 


\section{Resultados Obtidos}

$\mathrm{O}$ testes computacionais com o $M S P F$ foram realizados em duas etapas. Na primeira, a implementação se baseou nos resultados obtidos por TANG \& DENARDO (1987) e WIDMER (1991) no qual são abordados respectivamente os problemas de minimização do número de troca de ferramentas a partir de uma seqüência inicial fixa de partes e o de minimização do número de instantes de parada para a troca de ferramentas. Em uma segunda etapa, passou-se para o estudo do comportamento do modelo quando são privilegiadas, por meio de pesos, as parcelas da função objetivo.

\subsection{Testes Iniciais para Minimização do Número de Troca de Ferramentas e/ou Setups}

Os algoritmos $A M I G$ e TSS foram codificados em linguagem $C$, e todos os testes rodados em um $P C-486 / 100 M z$. TANG \& DENARDO (1987) considerando 10 ferramentas, 10 partes e a capacidade do magazine igual a 4 geraram 5 conjuntos de dados. YANASSE, VIJAYKUMAR \& OLIVO (1996) geraram aleatoriamente 100 conjuntos de dados com as mesmas características propostas por TANG \& DENARDO (1987). O MSPF foi submetido ao mesmo teste utilizando os mesmos dados utilizados por Yanasse, VIJAYKUMAR \& OLIVO (1996). A Tabela 1 mostra os resultados da aplicação aos dados dos algoritmos de Tang e Denardo (A), Yanasse, Vijaykumar e Olivo (B) e o $M S P F$, mostrando os números mínimos (mín.) e máximos (máx.) de trocas de ferramentas observadas e a média e o desvio padrão (desvio).

Os resultados mostrados na Tabela 1 são animadores. O modelo, embora de propósito mais geral, mostrou-se capaz de obter resultados melhores que heurísticas específicas para o problema de minimização do número de trocas de ferramentas.

WIDMER (1991) propôs uma heurística para a minimização do número de instantes de parada para a troca de ferramentas. Utilizando ao mesmos dados de TANG \& DENARDO (1987), WIDMER (1991) obteve 4 instantes de parada para a troca de ferramentas. Aplicando a heurística proposta por TANG \& DENARDO (1987) aos resultados obtidos, WIDMER obteve um mínimo de 8 trocas de ferramentas. O MSPF obteve um mínimo de 7 trocas de ferramentas e de 4 instantes de parada em uma solução não considerando uma seqüência inicial de partes fixas e mantendo a influência das demais parcelas da função objetivo.

\subsection{Análise de Políticas de Otimização para o MSFP}

Os resultados obtidos nesta fase foram considerados muito interessantes, mas não foram encontradas referências para comparação. Os resultados estão a disposição em GÓMEZ (1996). Os algoritmos AMIG e TSS foram inicialmente rodados 100 vezes com a seguinte matriz $T$ considerada anteriormente em TANG $\&$ DENARDO (1987).

ferramentas

\begin{tabular}{c|ccccccccc}
\multicolumn{1}{c}{$p a r t e s$} & $\mathbf{1}$ & $\mathbf{2}$ & $\mathbf{3}$ & $\mathbf{4}$ & $\mathbf{5}$ & $\mathbf{6}$ & $\mathbf{7}$ & $\mathbf{8}$ & $\mathbf{9}$ \\
$\mathbf{1}$ & 1 & 0 & 0 & 1 & 0 & 0 & 0 & 1 & 1 \\
$\mathbf{2}$ & 1 & 0 & 1 & 0 & 1 & 0 & 0 & 0 & 0 \\
$\mathbf{3}$ & 0 & 1 & 0 & 0 & 0 & 1 & 1 & 1 & 0 \\
$\mathbf{4}$ & 0 & 0 & 0 & 0 & 0 & 0 & 1 & 0 & 0 \\
$T=\mathbf{5}$ & 0 & 0 & 0 & 0 & 0 & 1 & 0 & 0 & 0 \\
$\mathbf{6}$ & 0 & 0 & 1 & 0 & 0 & 0 & 0 & 0 & 0 \\
$\mathbf{7}$ & 1 & 0 & 0 & 0 & 1 & 0 & 1 & 0 & 1 \\
$\mathbf{8}$ & 0 & 0 & 1 & 0 & 1 & 0 & 0 & 1 & 0 \\
$\mathbf{9}$ & 0 & 0 & 0 & 0 & 1 & 0 & 1 & 0 & 0 \\
$\mathbf{1 0}$ & 1 & 1 & 0 & 1 & 0 & 0 & 0 & 0 & 0
\end{tabular}


Tabela 1 - Resultados para o problema de minimização do número de trocas de ferramentas

\begin{tabular}{|l||c|c|c|c|}
\hline Heurísticas & Mín. & Máx. & Média & Desvio \\
\hline A & 6 & 13 & 9,45 & 1,22 \\
B & 6 & 12 & 8,39 & 1,31 \\
MSPF & 6 & 11 & 7,73 & 1,21 \\
\hline
\end{tabular}

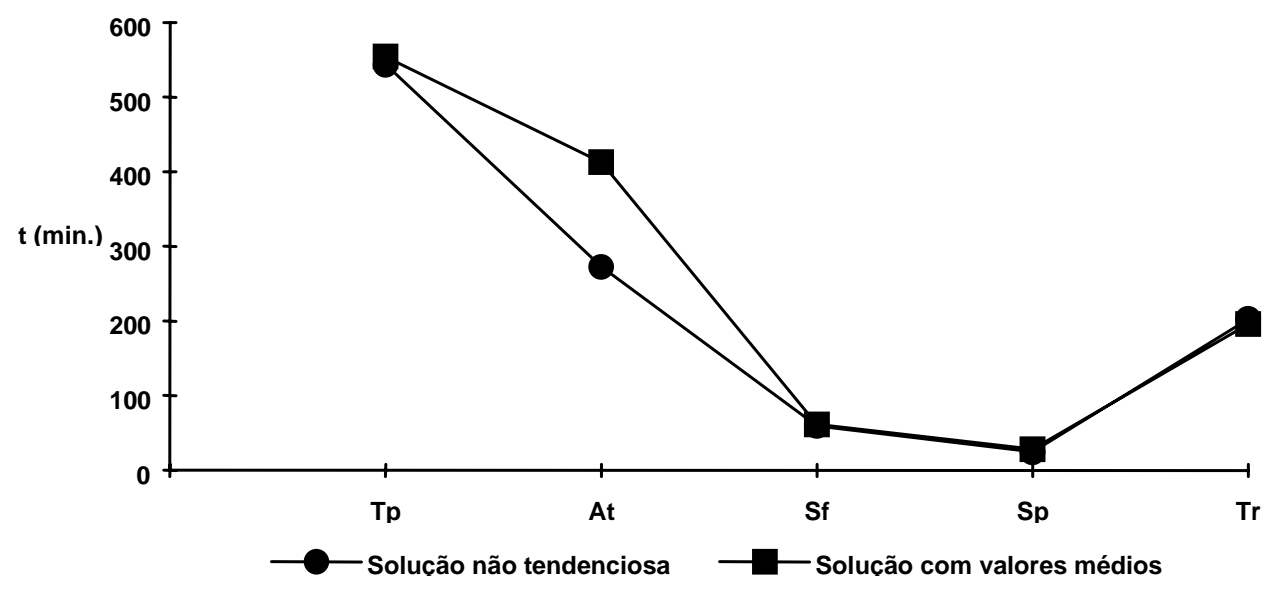

Figura 1 - Gráfico comparativo entre o comportamento médio do MSPF e a solução não tendenciosa

Os pesos da função objetivo foram variados aleatoriamente no intervalo [0,20], visando a representação de diferentes políticas. Não houve a preocupação em analisar o comportamento dos resultados obtidos dada a política associada. $\mathrm{O}$ objetivo foi gerar resultados que refletissem o comportamento do modelo frente a diferentes situações. Dos valores obtidos das parcelas da função objetivo para cada rodada (considerando nbmax igual a 20), foram derivadas as seguintes médias com os respectivos desvios padrões amostrais $(\sigma)$ :

- $T p$, tempo de produção: 554,27 min e $\sigma=3,87$

- At, tempo de atraso: 412,91 min. e $\sigma=17,51$;

- número de paradas para troca de ferramentas: 5,74 paradas $(28,73 \mathrm{~min})$ e $\sigma=0,56$ $(2,78)$;

- número de trocas de ferramentas: 11,45 ferramentas $(61,82 \mathrm{~min}$.) e $\sigma=0,96(3,89)$;

- $T r$, tempo restante dos turnos: $196,55 \mathrm{~min}$. e $\sigma=4,32$.
Foi observado que no pior caso na décima oitava iteração a melhor solução foi obtida. Sendo o número médio de iterações para obter a melhor solução igual a 7,5 iterações. De modo a obter uma solução em que nenhum dos tempos fosse privilegiado dividiu-se o tempo médio de produção pelos tempos médios das parcelas da função objetivo. Foram obtidas as seguintes relações para os pesos: $\mathrm{P}_{1} \propto \mathrm{P}_{2} .1,34 \propto \mathrm{P}_{3} .8 .97 \propto$ $\mathrm{P}_{4} .19,29 \propto \mathrm{P}_{5} .2,82$.

A Figura 1 compara o comportamento do modelo utilizando os valores médios e utilizando os valores obtidos com os pesos proporcionais, fazendo $P_{1}=10$. A melhor solução obtida com os pesos proporcionais foi denominada de solução não tendenciosa.

Considerando a solução não tendenciosa como parâmetro, o comportamento do sistema foi analisado para diferentes políticas de otimização. Para ilustrar a metodologia seguida, a seguir é mostrada uma análise para a política de minimização do número de ferramentas. 
Tabela 2 - Mimimização do número de trocas de ferramentas

\begin{tabular}{|c|cccccccccccccccc|}
\hline $\boldsymbol{P}_{\boldsymbol{4}}$ & 10 & 20 & 30 & 40 & 50 & 60 & 70 & 80 & 90 & 100 & 110 & 200 & 300 & 400 & 500 & 1000 \\
\hline $\boldsymbol{T} \boldsymbol{p}$ & 544 & 554 & 505 & 505 & 554 & 554 & 554 & 554 & 554 & 554 & 514 & 514 & 514 & 514 & 514 & 514 \\
\hline $\boldsymbol{A t}$ & 273 & 276 & 641 & 641 & 685 & 767 & 767 & 767 & 767 & 767 & 895 & 895 & 895 & 895 & 895 & 895 \\
\hline $\boldsymbol{S p}$ & 5 & 5 & 4 & 4 & 4 & 4 & 4 & 4 & 4 & 4 & 4 & 4 & 4 & 4 & 4 & 4 \\
\hline $\boldsymbol{S f}$ & 11 & 11 & 8 & 8 & 8 & 7 & 7 & 7 & 7 & 7 & 8 & 8 & 8 & 8 & 8 & 8 \\
\hline $\boldsymbol{T} \boldsymbol{r}$ & 203 & 203 & 220 & 220 & 220 & 224 & 224 & 224 & 224 & 224 & 220 & 220 & 220 & 220 & 220 & 220 \\
\hline
\end{tabular}

\subsubsection{Minimização do Número de Trocas de Ferramentas}

Dado que o objetivo é minimizar o número de trocas de ferramentas foi privilegiado somente o peso correspondente, $\mathrm{P}_{4}$. A Tabela 2 mostra alguns resultados fornecidos pelo $M S P F$ em que os pesos $\mathrm{P}_{1}, \mathrm{P}_{2}, \mathrm{P}_{3}$ e $\mathrm{P}_{5}$ são mantidos constantes com os valores correspondentes aos da solução não tendenciosa e $\mathrm{P}_{4}$ tem seu valor aumentado. Com exceção de $S f$ e $S p$, cujos valores na Tabela 2 representam o número de trocas de ferramentas e instantes de parada, respectivamente, a unidade dimensional das demais parcelas representativas da função objetivo é o minuto. Os resultados abaixo correspondem a 16 buscas tabu com nbmax igual a 20.

Observa-se na tabela citada que na faixa $60 \leq P_{4} \leq 100$ obteve-se o menor número de trocas de ferramentas: 7 . Um aumento significativo do tempo de atraso (de 273 para 895 minutos) confirma o conflito existente entre atender as datas de vencimento e procurar minimizar o número de trocas de ferramentas. Dado que $\mathrm{P}_{4}$ foi assumindo valores cada vez maiores, a influência dos valores das demais parcelas da função objetivo foram se tornando cada vez mais insignificantes. Deve ficar claro que cada vez que se altera o valor de um peso tem-se em verdade um novo contexto na função objetivo. $\mathrm{O}$ aumento de um peso significa que a parcela a que ele está designado vai ter maior influência na otimização desejada. Uma situação extrema seria aquela em que a magnitude do valor de $\mathrm{P}_{4}$ fosse tão grande em relação aos demais pesos, que o somatório obtido pelas demais parcelas da função objetivo não influenciasse mais na escolha dos melhores movimentos. Constata-se, portanto, que por meio de gerenciamento dos pesos pode-se restringir o tamanho da vizinhança nas buscas locais. Neste caso em particular, a medida que movimentos os conduzem a um maior número de trocas de ferramentas diminui a chance de serem escolhidos. Finalizando, a diminuição do número de instantes de parada para a troca de ferramentas $(S p)$ surge como decorrência natural. Observa-se também, que os tempos de produção e de períodos ociosos nos turnos não apresentaram grandes variações.

A Tabela 3 mostra a seqüência de partes e carregamento de ferramentas geradas pelo $M S P F$ quando $\mathrm{P}_{4}=10$.

A seqüência de partes obtida foi $s=((6),(3)$, (1), $(7,9,4),(2,8),(5,10))$. A seqüência das partes dentro dos lotes é determinada em ordem crescente em relação às datas de vencimento. Foram observados 5 instantes de parada e 11 trocas de ferramentas.

A Tabela 4 mostra a seqüência de partes e carregamento de ferramentas no magazine para $\mathrm{P}_{4}$ igual a 60.

A sequiência de partes obtida foi $s=((9,4,7)$, $(6,2,8),(1),(10),(3,5))$. Foram observados 4 instantes de parada e 7 trocas de ferramentas.

A Tabela 5 mostra a seqüência de partes e carregamento de ferramentas obtidos para $\mathrm{P}_{4}$ igual a 110.

Comparando-se as Tabelas 4 e 5 observa-se que o número de instantes de parada para troca 
Tabela 3 - Seqüência de partes e carregamento de ferramentas no magazine $(P 4=10)$

\begin{tabular}{|c|c|c|c|c|c|}
\hline Lotes & \multicolumn{4}{|c|}{ Ferramentas no magazine } & Número de trocas \\
\hline 6 & 2 & 3 & 6 & 7 & - \\
\hline 3 & 2 & 6 & 7 & 8 & 1 \\
\hline 1 & 1 & 4 & 8 & 9 & 3 \\
\hline $7,9,4$ & 1 & 5 & 7 & 9 & 2 \\
\hline 2,8 & 1 & 3 & 5 & 8 & 2 \\
\hline 5,10 & 1 & 2 & 4 & 6 & 3 \\
\hline \multicolumn{5}{|c|}{ No de $S p=5$} & No de $S f=11$ \\
\hline
\end{tabular}

Tabela 4 - Seqüência de partes e carregamento de ferramentas no magazine $\left(P_{4}=60\right)$

\begin{tabular}{|c|cccc|c|}
\hline Lotes & \multicolumn{4}{|c|}{ Ferramentas no magazine } & Número de trocas \\
\hline $9,4,7$ & 1 & 5 & 7 & 9 & - \\
$6,2,8$ & 1 & 3 & 5 & 8 & 2 \\
1 & 1 & 4 & 8 & 9 & 2 \\
10 & 1 & 2 & 4 & 8 & 1 \\
3,5 & 2 & 6 & 7 & 8 & 2 \\
\hline \multicolumn{2}{|r}{$N o d e S p=4$} \\
\hline
\end{tabular}

Tabela 5 - Sequiência de partes e carregamento de ferramentas no magazine ( $P 4=110)$

\begin{tabular}{|c|c|c|c|c|c|}
\hline Lotes & \multicolumn{4}{|c|}{ Ferramentas no magazine } & Número de trocas \\
\hline $3,4,5$ & 2 & 6 & 7 & 8 & - \\
\hline $6,2,8$ & 1 & 3 & 5 & 8 & 3 \\
\hline 9,7 & 1 & 5 & 7 & 9 & 2 \\
\hline 10 & 1 & 2 & 4 & 9 & 2 \\
\hline 1 & 1 & 4 & 8 & 9 & 1 \\
\hline \multicolumn{5}{|c|}{ No de $S p=4$} & No de $S f=8$ \\
\hline
\end{tabular}

de ferramentas é igual a 4, mas o número de trocas de ferramentas na Tabela 5 é igual a $8 . \mathrm{Ou}$ seja, o número de instantes de parada é o mesmo mas o número de trocas de ferramentas variou. Também deve ser observado que as sequiências das partes e de carregamento de ferramentas nos exemplos são diferentes. A seqüência de partes obtida foi $s=((3,4,5),(6,2,8),(9,7),(10),(1))$.

\subsubsection{Desconsiderando o Atraso}

De modo a anular a influência do atraso no problema de minimização do número de trocas de ferramentas foram realizados os experimentos mostrados na Tabela 6. Os pesos referentes aos tempos de atraso e dos períodos ociosos dos turnos são anulados. Nos demais pesos, com exceção de $\mathrm{P}_{4}$, é mantida a proporcionalidade da 
Tabela 6 - Minimização do número de trocas de ferramentas desconsiderando o atraso

\begin{tabular}{|l|cccccccc|}
\hline $\boldsymbol{P}_{\boldsymbol{4}}$ & 8,97 & 100 & 500 & 600 & 650 & 680 & 700 & 1000 \\
$\boldsymbol{T} \boldsymbol{p}$ & 540 & 520 & 520 & 520 & 520 & 514 & 514 & 514 \\
$\boldsymbol{A t}$ & 1034 & 1232 & 1232 & 1232 & 1232 & 895 & 895 & 895 \\
$\boldsymbol{S p}$ & 4 & 4 & 4 & 4 & 4 & 4 & 4 & 4 \\
$\boldsymbol{S f}$ & 7 & 7 & 7 & 8 & 8 & 8 & 8 & 8 \\
$\boldsymbol{T r}$ & 224 & 224 & 224 & 224 & 224 & 220 & 220 & 220 \\
\hline
\end{tabular}

solução não tendenciosa. Os dados da Tabela 6, com exceção aos referentes a $S p$ e $S f$ que representam número de instantes de parada e o número de trocas de ferramentas, estão em minutos.

Analisando a Tabela 6, observa-se que enquanto o número de trocas de ferramentas apresenta seu menor valor o comportamento do atraso é crescente ou constante em um patamar superior. Quando o número de trocas de ferramentas aumenta o atraso declina e estabiliza em um patamar inferior. Conclui-se portanto que existe uma forte relação de natureza inversa entre o tempo de atraso e a minimização do número de ferramentas. Este fato confirma a expectativa do modelo em relação ao conflito existente entre o atendimento das datas de vencimento e das abordagens de seleção de partes.

\section{Conclusões}

$\mathrm{O}$ modelo de seleção de partes e de ferramentas (MSPF) apresentado neste artigo permite, a partir de uma seqüência inicial, obter várias sequiências de partes e carregamento de ferramentas que refletem as estratégias determinadas pelos pesos designados às parcelas da função objetivo. Além disto, o modelo permite

\section{Referências Bibliográficas}

BARD, J.F.: "A heuristic for minimization the number of tool switches on a flexible machine". IIE Transactions, 20 (4): 382-391, dez., 1988. que os problemas de seleção de partes e de carregamento de ferramentas sejam abordados conjuntamente. Este é um ponto inovador. Todas as heurísticas desenvolvidas atualmente para resolver estes problemas são dedicadas a soluções individuais dos mesmos. Na solução do problema de "scheduling" com restrições ficou patente o conflito existente entre tentar gerar uma sequiência de partes que minimize o atraso e ao mesmo tempo que atenda as abordagens de seleção de partes e de carregamento de ferramentas. Não existe solução sem conflito para o problema do atraso. Ou privilegia-se a minimização do número de trocas de ferramentas e de instantes de parada para a troca de ferramentas ou administra-se o conflito pelo gerenciamento dos pesos das parcelas da função objetivo. $\mathrm{O}$ modelo fornece, também, vários resultados que podem ser utilizados como referência para outros estudos que abordem os problemas de seleção de partes, carregamento de ferramentas e de "scheduling" com restrições.

\section{Agradecimentos}

O trabalho do segundo autor recebeu apoio parcial do $\mathrm{CNPq}$ através dos projetos de números 520844/96-3, 680082/95-6 e da FAPESP no projeto número 95/9522-0.

BLAZEWICZ, J.; EISELT, H.; FINKE, G.; LAPORTE, G. \& WEGLARZ, J.: "Scheduling tasks and vehicles in a flexible manufacturing systems". International Journal FMS, 4: 5-16, 1991. 
BLAZEWICZ, J. \& FINKE, G.: "Scheduling with resource management in manufacturing systems". European Journal of Operational Research, $\underline{76}$ : 1-14, 1994.

CRAMA, Y.; KOLEN, A.W.J.; OERLEMANS, A.G. \& SPIEKSMA, F.C.R.: "Minimizing the number of tool switches on a flexible machine". The International Journal of FMS, 1: 33-55, 1994.

FOLLONIER, J.P.: "Minimization of the number of tool switches on a flexible manufacturing machine". Belgian Journal of Operations Research, Statistics and Computer Science, 34 (1): 55-72, 1994.

GÓMEZ, A.T.: "Modelo para sequenciamento de partes e ferramentas em um sistema de manufatura flexível com restrições as datas de vencimento e a capacidade do magazine". Tese de Doutorado em Computação Aplicada - Instituto Nacional de Pesquisas Espaciais, São José dos Campos, 1996.

GLOVER, F. \& LAGUNA, M.: "Tabu Search". In Modern Heuristic Techniques for Combinatorial Problems, Ed. Colin E. Reeves. MacGraw-Hill. p.70-150, 1995.

GYAMPAH, K.: "A comparative study of FMS tool allocation and part type selection approaches for varying part type mix". The International Journal of FMS, ㅁ: 179-207, 1994.

HE, W. \& KUSIAK, A.: "A scheduling manufacturing systems". Computers in Industry, 20: 163-175, 1992.

HIRABAYASHI, R.; SUZUKI, H. \& TSUCHIYA, N.: "Optimal tool module design problem for $\mathrm{NC}$ machine tools". Journal of the Operations Research Society of Japan, 27 (3): 205-229, set., 1984.

HWANG, S.S. \& SHOGAN, W.: "Modeling and solving an FMS part selection problem". International Journal of Production Research, 27 (8): 1349-1366, 1989.

JAIKUMAR, R. \& VAN WASSENHOVE, L.N.: "A production planning framework for flexible manufacturing systems". Journal of Manufacturing and Operations Management, (2): 52-79, 1989.

KOUVELIS, P.: "Design and planning problems in flexible manufacturing systems: A critical review". Journal of Intelligent Manufacturing, $\underline{3}$ (2): 75-100, 1992.
KUSIAK, A. \& CHOW, W.S.: "Efficient solving of the group technology problem". Working Paper 686. University of Manitoba, Department of mechanical engineering, Winnipeg, Manitoba, 1986.

MORENO, A.A. \& DING, F.: "Heuristics for the FMS-loading and part-type selection problems". The International Journal of Flexible Manufacturing Systems, 5: 287-300, 1993.

OERLEMANS, A.G.: "Flexible manufacturing: concepts and methods". JORBEL - Belgian Journal of Operations Research, Statistics and Computer Science, $\underline{34}$ (1): 5-22, 1994.

STECKE, K.E.: "Algorithms for efficient planning and operation of a particular FMS". The International Journal of Flexible Manufacturing Systems, (1): 287-327, 1989.

STECKE, K.E. \& KIM, I.: "A study of FMS part type selection approaches for short-term production planning". The International Journal of Flexible Manufacturing Systems, 1 (1): 7-29, 1988.

STECKE, K.E. \& KIM, I.: "A flexible approach to part type selection in flexible flow systems using part mix ratios". International Journal of Production Research, 29 (1): 53-57, 1991.

STECKE, H.E. \& TOCZYLOWSKI, E.: "Profitbased FMS dynamic part type selection over time mid-term production planning". European Journal of Operational Research, (63): 54-65, 1992.

TANG, C.S. \& DENARDO, E.V.: "Models arising from flexible manufacturing machine, part I: minimization of the number of tool switches". Operations Research, $\underline{36}$ (5): 767-776, 1987.

TANG, C.S. \& DENARDO, E.V.: "Models arising from flexible manufacturing machine, part II: minimization of the number of switches instants". Operations Research, $\underline{36}$ (5): 778-784, 1988.

WIDMER, M.: "Job shop scheduling with tooling constraints: a tabu search approach". Journal of Operational Research Society, 42 (1): 75-82, 1991. 
YANASSE, H.H.; VIJAYKUMAR, N.I. \& OLIVO, A.:

"A heuristic procedure to minimize the number of tool switches in a flexible manufacturing machine". In: XXVIII Simpósio Brasileiro de
Pesquisa Operacional, Rio de Janeiro, 1996. Anais, Rio de Janeiro, SOBRAPO, v.6, p.599-603, 1996.

\title{
A FLEXIBLE MANUFACTURING SYSTEM MODEL WHICH CONSIDERS CONSTRAINTS OF TIME AND MAGAZINE CAPACITY
}

\begin{abstract}
In this paper we propose a model for scheduling parts and tools in a flexible manufacturing system composed of one machine. We consider the due dates of the parts to be processed, production periods and the physical capacity constraints of the magazine which stores the tools required for processing the parts. The model considers the problems of part selection, tool loading and part scheduling using a tabu search approach. An initial algorithm is proposed to identify clusters of parts and tools taking into consideration the magazine capacity. The tabu search optimization function indicates some possible optimization policies determined by weighted objective functions. Several tests were conducted to validate the model based on four optimization policies: minimization of the number of tool changes, the number of times the machine is stopped for tool changes, the delay, and the idle periods during production shifts.
\end{abstract}

Key words: part selection, tool loading, scheduling. 